\title{
Erratum to: FGFR1 expression and gene copy numbers in human lung cancer
}

\section{Lukas H. Kohler • Masoud Mireskandari • \\ Thomas Knösel • Annelore Altendorf-Hofmann • \\ Almut Kunze • Andreas Schmidt • Norbert Presselt • \\ Yuan Chen • Iver Petersen}

Published online: 7 August 2012

(C) Springer-Verlag 2012

\section{Erratum to: Virchows Arch (2012) 461:49-57 \\ DOI 10.1007/s00428-012-1250-y}

There was an error in the original version of Fig. 7 representing the DNA imbalances of the 4 lung cancer subtypes as detected by conventional CGH. For large cell lung cancer (LCLC), the ideogram of chromosome
9 together with the histograms of DNA gains and losses was shown and not chromosome 8 harbouring the FGFR1 locus.

The corrected version with the chromosome 8 histogram (see new figure), however, indicates an identical finding, i.e. that chromosome $8 \mathrm{p}$ is more often affected by deletions than DNA gains.

The online version of the original article can be found at http://dx.doi.org/ 10.1007/s00428-012-1250-y.

L. H. Kohler · M. Mireskandari · T. Knösel · Y. Chen •

I. Petersen $(\triangle)$

Institute of Pathology, Jena University Hospital,

Friedrich-Schiller-University,

Ziegelmuehlenweg 1,

07740 Jena, Germany

e-mail: Iver.Petersen@med.uni-jena.de

\section{A. Altendorf-Hofmann}

Department of General and Visceral and Vascular Surgery,

Jena University Hospital, Friedrich-Schiller-University,

Erlanger Allee 101,

07740 Jena, Germany

A. Kunze $\cdot$ A. Schmidt

Department of Pathology, Central Clinic Bad Berka,

Robert-Koch-Allee 9,

99438 Bad Berka, Germany

N. Presselt

Department of Thoracic and Vascular Surgery,

Central Clinic Bad Berka,

Robert-Koch-Allee 9,

99437 Bad Berka, Germany

\section{Present Address:}

T. Knösel

Institute of Pathology, Ludwig-Maximilian-University,

Thalkirchner Strasse 36,

80337 Munich, Germany 


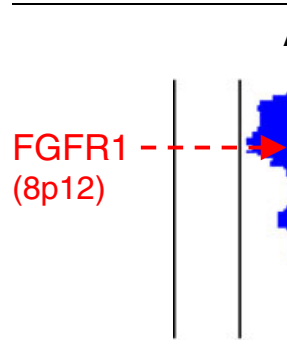

$100 \% 50 \%$
ADC

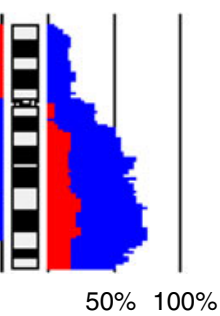

SCC

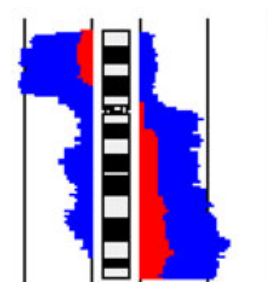

LCLC

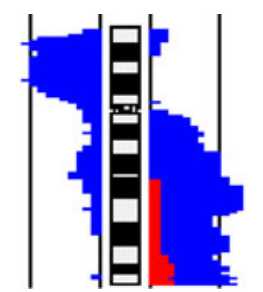

SCLC

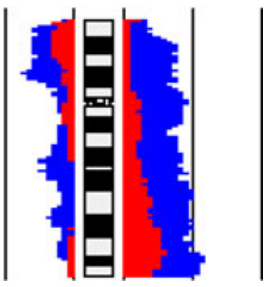

Fig. 7 DNA imbalances of lung cancer subtypes according to conventional comparative genomic hybridization/CGH (compiled from data of Petersen I in Travis et al. 2004, [30]). Cases with DNA gains were represented by the histogram on the right side of the chromosome

ideogram, cases with DNA loss on the left side. The data indicated that $8 \mathrm{p}$ deletions are at least as frequent as DNA gains with pronounced gains (indicated by the red colour) as putative correlates of an amplification being undetectable in the NSCLC subtypes 